\title{
Pleiotropic effects of schizophrenia- associated genetic variants in neuron firing and cardiac pacemaking revealed by computational modeling
}

Tuomo Mäki-Marttunen (1) ${ }^{1,2}$, Glenn T. Lines², Andrew G. Edwards², Aslak Tveito², Anders M. Dale 3,4,5, Gaute T. Einevoll ${ }^{6,7}$ and Ole A. Andreassen (10)

\begin{abstract}
Schizophrenia patients have an increased risk of cardiac dysfunction. A possible factor underlying this comorbidity are the common variants in the large set of genes that have recently been discovered in genome-wide association studies (GWASs) as risk genes of schizophrenia. Many of these genes control the cell electrogenesis and calcium homeostasis. We applied biophysically detailed models of layer $V$ pyramidal cells and sinoatrial node cells to study the contribution of schizophrenia-associated genes on cellular excitability. By including data from functional genomics literature to simulate the effects of common variants of these genes, we showed that variants of voltage-gated $\mathrm{Na}^{+}$channel or hyperpolarization-activated cation channel-encoding genes cause qualitatively similar effects on layer $\vee$ pyramidal cell and sinoatrial node cell excitability. By contrast, variants of $\mathrm{Ca}^{2+}$ channel or transporter-encoding genes mostly have opposite effects on cellular excitability in the two cell types. We also show that the variants may crucially affect the propagation of the cardiac action potential in the sinus node. These results may help explain some of the cardiac comorbidity in schizophrenia, and may facilitate generation of effective antipsychotic medications without cardiac side-effects such as arrhythmia.
\end{abstract}

\section{Introduction}

Schizophrenia (SCZ) is a heritable mental disorder with a high burden of morbidity and large social impacts ${ }^{1}$. A recent genome-wide association study (GWAS) has identified more than a hundred genetic loci exceeding genome-wide significance ${ }^{2}$. The loci implicate genes that encode numerous ion channel subtypes and calcium transporters and are major contributors to the functions of cells in not only brain but also organs outside the central nervous system, such as heart. Evidence for increased cardiac dysfunction in SCZ patients are shown

\footnotetext{
Correspondence: Tuomo Mäki-Marttunen (tuomomm@uio.no)

${ }^{1}$ NORMENT, KG Jebsen Centre for Psychosis Research, Institute of Clinical

Medicine, University of Oslo, Oslo, Norway

${ }^{2}$ Simula Research Laboratory and Center for Cardiological Innovation, Oslo,

Norway

Full list of author information is available at the end of the article
}

by meta-studies that reported a $2.5-3$-fold increase in mortality rates ${ }^{3,4}$. Approximately $40 \%$ of the excess deaths are caused by accidents and suicides, while the remaining $60 \%$ are natural ${ }^{5}$ - and largely due to cardiovascular disease $^{6}$. Some of these excess deaths are linked to the increased risk of sudden cardiac death conveyed by longterm use of antipsychotic drugs ${ }^{7}$, many of which are known to have side-effects related to arrhythmia, including prolongation of QT interval ${ }^{8}$ and torsades de pointes ${ }^{9}$. In parallel to these observations, GWASs of cardiac phenotypes, such as electrocardiographic (ECG) measures, highlight a set of genes that overlaps with the one discovered in GWASs of SCZ ${ }^{10,11}$. Nevertheless, both the genetic and mechanistic connections between cardiac and neural phenotypes in SCZ patients remain poorly understood. Here, we attempt to combine our recently developed genetic approaches with biophysical models of 
well-characterized cardiac and neuronal cell types to provide general mechanistic links between neural and cardiac tissue for $\mathrm{SCZ}$-associated variants.

It is of key relevance to know if there is an inherent, genetic risk in addition to the external, drug-induced risks in the treatment of SCZ that may underlie the comorbidity between cardiac disease and SCZ-the SCZassociated single-nucleotide polymorphisms (SNPs) might, however, as well be protective against cardiac disease. The effects of primarily brain disorder-related SNPs on cardiac phenotypes is a largely unexplored area, while there are a few examples of the opposite: SNPs that were first identified by a cardiac disease and then found to convey a risk of brain dysfunction, such as seizures ${ }^{12,13}$. The cellular homogeneity in the heart, in contrast to the heterogeneity in both structure and function of the brain, is an important aid in uncovering cross-tissue functional genetics in both approaches.

SCZ is associated with genes affecting transmembrane currents of all major cationic species, $\mathrm{Na}^{+}, \mathrm{K}^{+}$, and $\mathrm{Ca}^{2+}$ (ref. 2). In addition, some of the SCZ-linked genes are involved in regulation of intracellular $\mathrm{Ca}^{2+}$ dynamics ${ }^{2}$, which importantly modulate cellular excitability in both the heart and brain, via a range of $\mathrm{Ca}^{2+}$-sensitive plasma membrane current carriers. The rise of biophysical modeling of neurons ${ }^{14}$ and cardiac pacemaker cells ${ }^{15}$ provide a solid basis for analyzing this intrinsic excitability as a coordinated interplay of ion channels and ion transporters. In addition, there is an increasing amount of in vitro data on the effect of genetic variations on such ion channel or calcium transporter functions, and much of these data can be implemented in the biophysical models. This opens the door for a mechanistic analysis of SCZrelated genes ${ }^{16}$, mapping the functional genomics data to predictions of cellular function and dysfunction both in neural and cardiac tissue.

In this work, we use computational modeling to study the contribution of SCZ-associated genes to cardiac and neuronal excitability. We focus our analyses on two wellstudied cell types that are central to cortical information processing and cardiac pacemaking, namely, layer $\mathrm{V}$ pyramidal cells (L5PCs) in the cortex and sinoatrial node cells (SANCs) in the myocardium. The apical tuft of an L5PC integrates non-local synaptic inputs, and is considered a biological substrate for cortical associations providing high-level context for low-level (e.g., sensory) inputs that arrive at the perisomatic compartments ${ }^{17}$. Therefore, the ability of L5PC to integrate the apical and perisomatic inputs has been proposed as one of the mechanisms that could be impaired in hallucinating patients ${ }^{17}$. The SANCs, in turn, have a key role in controlling heart rate as the primary pacemakers of the mammalian heart. While SANCs derive from cardiac lineage and are, therefore, regarded as a specialized form of myocardium, their morphology, electrophysiology, and ion-channel expression profiles are the most neuron-like of any studied cardiac muscle cell type. As such, they represent a population of cardiac cells that may be most apt to display functional alterations as a result of SCZ-associated variants. We apply two recent L5PC models $^{18,19}$ and two recent SANC models ${ }^{20,21}$ to argue for the generality of our findings.

We show that subtle SNP-like variants of ion-channel and calcium-transporter-encoding genes cause notable effects in intrinsic excitability of both neurons and heart cells. Our approach is limited by the data concerning the functional effects of the SCZ-related common genetic variants. To overcome this limitation, we concentrate on a set of in vitro-observed effects of more extreme genetic variations, as described previously ${ }^{16}$. A key assumption of our approach is that the effects of SNP variants can be represented as downscaled versions of the more extreme variants, and that the emergence of disease phenotypes results from the combined effect of a large number of subtle SNP effects ${ }^{22,23}$. Our results contribute to explaining some of the cardiac comorbidity in schizophrenia, and could form the basis for development of antipsychotic medications that are free from cardiac side-effects.

\section{Materials and methods}

\section{Models of neurons and cardiac pacemaker cells}

We apply two multicompartmental L5PC models, "Hay"18 and "Almog"19 model, and two single-compartment SANC models, "Kharche"20 and "Severi" ${ }^{21}$ model. These models include Hodgkin-Huxley type description for channel activation and inactivation, and hence, changes related to certain ion-channel-encoding gene variants that have been observed in experiments can be directly attributed to a change of one or more parameters of these models. The Hay and Almog neuron models are based on electrophysiological recordings and cell stainings from rat neocortical slices, while the Kharche and Severi models are based on mouse and rabbit data, respectively. For details on the models, see Supplementary information.

Both L5PC models are simulated using NEURON software and Python interface using adaptive time-step integration. The 0-dimensional (point-cell) and 1dimensional (chain of cells) SANC models are simulated using MATLAB, and the numerical integration is carried out using the variable time-step, stiff differential equation solver ode15s (0-dimensional) or the finite difference method with $0.01 \mathrm{~ms}$ time step (1-dimensional). For the 2dimensional problem, we use the monodomain model ${ }^{24}$, which is simulated using the finite element method solver FEniCS $^{25}$. For details on the spatial distribution of parameters in the $2 \mathrm{D}$ simulations, see ref. ${ }^{26}$. Scripts for running L5PC and OD SANC simulations are publicly 
available (https://senselab.med.yale.edu/ModelDB/show Model.cshtml?model=187615).

\section{Genes included in the study}

We chose the set of SCZ-associated genes as follows: We used the SNP-wise $p$-value data of ref. 2, and for each gene of interest, determined the minimum $p$-value among those SNPs that were located in the considered gene. We performed this operation for all genes encoding either subunits of voltage-gated $\mathrm{Ca}^{2+}, \mathrm{K}^{+}$, or $\mathrm{Na}^{+}$channels, subunits of an SK, leak, or hyperpolarization-activated cyclic nucleotide-gated $(\mathrm{HCN})$ channel, or $\mathrm{Ca}^{2+}$-transporting ATPases. Several of these genes were found to contain SNPs bearing a high risk of SCZ ( $p$-value smaller than $3 \times 10^{-8}$ in the data of ref. 2, namely, CACNA1C, $C A C N B 2, C A C N A 1 I, A T P 2 A 2$, and HCN1. Using a more relaxed threshold ( $p$-value smaller than $3 \times 10^{-5}$ ) extended this set by the genes CACNA1D, SCN1A, SCN9A, KCNN3, KCNS3, KCNB1, KCNMA1, and ATP2B2. This selection was identical to that in our previous work ${ }^{16}$. In this work, however, we concentrate on the genes that are likely to play a role in both L5PCs and SANCs: these are CACNA1C, CACNB2, CACNA1I, ATP2A2, HCN1, CAC$N A 1 D$, and SCN1A.

It should be noted that we used the SNPs reported in ref. 2 only to name the above SCZ-related genes, and due to lack of data on their electrophysiological effects, we could not include the actual SCZ-related SNPs. In fact, only 14 of 527 SNPs that had a $p$-value smaller than $3 \times$ $10^{-5}$ were highlighted in online databases (PubMed or SNPedia), and none of these 14 SNPs have yet been studied functionally, either in native or in heterologous cells. Therefore, we searched in PubMed for functional genomic studies reporting the effects of any genetic variant of the above genes, as described below. We only included studies that reported electrophysiological or intracellular $\mathrm{Ca}^{2+}$ imaging data, but we accepted studies performed using all tissue types. Nevertheless, most of the included studies were carried out in embryonic kidney cells.

\section{Gene variants and their downscaled versions}

Table 1 lists all studies ${ }^{27-56}$ that we found where the effects of a variant were measured in a way that could be directly implemented as a parameter change in our models. A more detailed version of this table is given in Supplementary information, Supplementary Table S1. As SCZ is a polygenic disorder, it has been proposed that the disorder will not be induced by any of the SCZ-related SNPs alone, but only when sufficiently many of them are represented. Furthermore, as most of these SNPs are common variants ${ }^{57}$, it is likely that none of them alone can cause radical cardiac dysfunction-however, their combination could underlie the risk of heart disease that has been observed in SCZ patients. This paradigm was
Table 1 Table of the genetic variants used in this study

\begin{tabular}{|c|c|c|c|}
\hline Gene & Refs. & Type of variant & Cell type \\
\hline CACNAIC & 27 & $\begin{array}{l}\text { L429T, L434T, S435T, S435A, } \\
\text { S435P }\end{array}$ & TSA201 \\
\hline CACNATC & 27 & L779T, I781T, I781P & TSA201 \\
\hline CACNATC & 28 & $\begin{array}{l}\text { G432X, A780X, G1193X, } \\
\text { A1503X }\end{array}$ & TSA201 \\
\hline CACNAIC & 29 & $\begin{array}{l}\text { I781X, C769P, G770P, N771P, } \\
\text { I773P, F778P, L779P, A780P, } \\
\text { A782P, V783P }\end{array}$ & TSA201 \\
\hline CACNATC & 30 & I781T, N785A, N785G, N785L & TSA201 \\
\hline CACNAIC & 31 & $\begin{array}{l}\text { Splice variants a1C77-A, -B, } \\
-C \text {, and -D }\end{array}$ & TSA201 \\
\hline CACNA1D & 32,33 & Splice variant 42A & TSA201/HEK293 \\
\hline CACNAID & 32,33 & Splice variant 435 & TSA201/HEK293 \\
\hline CACNAID & 34,35 & Homozygous knockout & $\begin{array}{l}\text { AV node cells / } \\
\text { chromaffin cells }\end{array}$ \\
\hline CACNAID & 36 & A749G & TSA201 \\
\hline CACNAID & 37 & V259D, I750M, P1336R & TSA201 \\
\hline CACNA1D & 38 & $\begin{array}{l}\text { rCav } 1.3 \mathrm{scg} \text { variant and } \\
\text { related mutants }\end{array}$ & TSA201 \\
\hline CACNB2 & 39 & $\mathrm{~T} 111$ & TSA201 \\
\hline CACNB2 & 40 & A1B2 vs A1 alone & HEK293 \\
\hline CACNB2 & 41 & $\begin{array}{l}\text { Splice variants N1, N3, N4, } \\
\text { N5 }\end{array}$ & HEK293 \\
\hline CACNB2 & 42 & D601E & TSA201 \\
\hline CACNA1I & 43 & $\begin{array}{l}\text { Alternative splicing of exons } \\
9 \text { and } 33\end{array}$ & HEK293 \\
\hline CACNA1I & 44 & $\begin{array}{l}\text { Truncated cDNAs L4, L6, } \\
\text { and L9 }\end{array}$ & HEK293 \\
\hline ATP2A2 & 45,46 & Heterozygous null mutation & $\begin{array}{l}\text { Myocytes, } \\
\text { embryonic } \\
\text { stem cells }\end{array}$ \\
\hline ATP2A2 & 47 & $\begin{array}{l}\text { Dairier's disease related } \\
\text { mutants }\end{array}$ & HEK293 \\
\hline ATP2A2 & 48 & $\begin{array}{l}\text { Dairier's disease related } \\
\text { mutants }\end{array}$ & HEK293 \\
\hline SCN1A & 49 & Q1489K & $\begin{array}{l}\text { Cultured } \\
\text { neocortical cell }\end{array}$ \\
\hline SCN1A & 50 & L1649Q & TSA201 \\
\hline SCNIA & 51 & $\mathrm{R} 859 \mathrm{H}$ & TSA201 \\
\hline SCN1A & 51 & R865G & TSA201 \\
\hline SCN1A & 52 & $\mathrm{~T} 1174 \mathrm{~S}$ & TSA201 \\
\hline SCNIA & 53 & M145T & TSA201 \\
\hline HCN1 & 54 & D135W, D135H, D135N & HEK293 \\
\hline HCN1 & 55 & $\begin{array}{l}\text { E229A, K230A, G231A, } \\
\text { M232A, D233A, S234A, } \\
\text { E235G, V236A, Y237A, } \\
\text { EVY235-237DDD }\end{array}$ & Oocytes \\
\hline HCN1 & 56 & WAG-HCN1 & Oocytes \\
\hline
\end{tabular}

For more details, see Supplementary Table S1 and Supplementary Table S2

used in this study in a similar fashion as in ref. 16. If the variants described in Supplementary Table S1 altered the neural response or cardiac pacemaking too dramatically (see conditions A1-A5 and B1-B2 in Supplementary 
information), the changes in the model parameters were brought closer to zero (all in proportion) until a point $c$ $\in[0,1]$ where one or more of the scaling conditions were first violated. This downscaling was performed so that those parameters that may receive both negative and positive values were scaled linearly $(\Delta \rightarrow c \Delta$, where $\Delta$ denotes the original increment to the underlying parameter as obtained from the literature data) while the parameters that receive only positive values were scaled on the logarithmic scale $\left(\Gamma \rightarrow \Gamma^{c}\right.$, where $\Gamma$ denotes the original factor of the underlying model parameter). In other words, the differences in offset and reverse potentials $\left(V_{\text {offm }}, V_{\text {offh }}\right)$ between control and variant neuron were expressed as an additive term $( \pm x \mathrm{mV})$, and this term $x$ was multiplied by a parameter $c$ in the downscaling procedure. By contrast, the differences in all the other model parameters $\left(V_{\text {slo }}, \tau, P_{\text {up }}, \gamma\right)$ between control and variant neuron were expressed as a multiplication $(\times x)$, where the downscaling caused this factor $x$ to be exponentiated by the same parameter $c$.

The scaling conditions were designed such that the variant L5PCs and SANCs retain their baseline firing (L5PC) and pacemaking (SANC) behavior: Conditions A1-A3 require that the variant L5PCs respond with the same numbers of spikes to certain stimuli as the control L5PCs, while conditions $\mathrm{A} 4$ and $\mathrm{B} 1$ require that the firing (L5PC) or pacemaking (SANC) frequency is not radically changed, and conditions A5 and B2 make sure that the shape of the action potentials is not too different from that of the control cell.

The downscaling was done separately for each applied model; see Supplementary Table S2 for the scaling parameters $c$ obtained for each variant and each model. Supplementary Fig. S1 illustrates the distribution of the variant effects of a single gene, $S C N 1 A$, on activation and inactivation voltage-dependency parameters in the Hay model, and shows that the variants span a large array of possible alterations of ion-channel dynamics. This is true also for the variants of other ion-channel-encoding genes (data not shown).

In the following, we present simulation data from the cells implemented with variants of different magnitude and direction, parametrized by variable $\epsilon$. This parametrization is done so that the final effect sizes of the variants are the values of Supplementary Table S2 multiplied or exponentiated by $\epsilon c$ (see Supplementary information). Variants with $\epsilon=\frac{1}{2}$ and $\epsilon=\frac{1}{4}$ mean that the variant effects on model parameters are half or quarter, respectively, of those of the threshold variants-these variants are, therefore, confirmed to obey the above-mentioned scaling conditions. In addition, we consider the variants $\epsilon=-\frac{1}{2}$ and $\epsilon=-\frac{1}{4}$, which represent parameter changes that are opposite to those of $\epsilon=\frac{1}{2}$ and $\epsilon=\frac{1}{4}$ variants.

\section{Results}

Pleiotropic effects of $\mathrm{Na}^{+}$and non-selective ion channel gene variants are analogous

To characterize the joint implications of SCZ-related genes on neuron excitability and cardiac pacemaking, we started by analyzing the effects of the downscaled versions of genetic variants of $\mathrm{Na}^{+}$and hyperpolarization-activated cyclic nucleotide-gated $(\mathrm{HCN})$ channel-encoding genes, namely, SCN1A and HCN1. Fig. 1a shows the time course of the membrane potential for several versions of a SCN1A variant, predicted by both L5PC models (when a somatic DC stimulus is applied) and both SANC models (at steady pacemaking). Moreover, Fig. $1 \mathrm{~b}$ shows the $\mathrm{f}-\mathrm{I}$ curves, where the firing frequency is plotted against the amplitude of the injected current, and threshold currents for inducing a spike in an L5PC for several SCN1A and $H C N 1$ variants, and the changes these variants cause to the pacemaking frequencies in SANCs.

The results of Fig. 1 show that the changes in $\mathrm{Na}^{+}$or HCN channels mostly caused similar effects in L5PCs and SANCs in terms of excitability. If the variant effect was excitatory (lower threshold of firing or steeper slope of $\mathrm{f}-\mathrm{I}$ curve, i.e., higher gain) in L5PCs, usually the effect in SANCs was excitatory as well (faster pacemaking). We confirmed this observation by changing only one model parameter at a time (see Supplementary Fig. S2). However, the effects of $\mathrm{Na}^{+}$channel variants on steady-state firing of the Hay-model neuron were usually small and often opposite to the corresponding effects in the Almogmodel neuron. Exceptions to the above-mentioned trend arise also in $\mathrm{HCN}$ channel variants that affect both the threshold and slope of activation, leading to diverse effects in the two L5PC models (see Supplementary information).

\section{Pleiotropic effects of variants of $\mathrm{Ca}^{2+}$ channel or transporter-encoding genes are non-analogous}

Next, we analyzed the implications of the genes that encode subunits of voltage-gated $\mathrm{Ca}^{2+}$ channels $(C A C$ NA1C, CACNA1D, CACNB2, CACNA1I) or $\mathrm{Ca}^{2+}$ transporters (ATP2A2). In Fig. 2, a representative set of variants of these genes is picked, and their effects on L5PC and SANC behavior is illustrated. In a similar fashion as in Fig. 1, Fig. 2a shows the time course of the membrane potential for different versions of one variant, and Fig. 2b shows the $\mathrm{f}-\mathrm{I}$ curves and pacemaking frequencies for several variants. A general trend is that variants that increase the pacemaking rhythm in SANCs decrease the firing frequency in L5PCs, and vice versa. This is supported by Fig. 3 showing the mean (averaged over the stimulus amplitudes of Fig. 1) firing frequencies of all implemented variants for the L5PC models and the corresponding pacemaking frequencies for the SANC models, and by Supplementary Figs. S3 and S4 showing results from single-parameter variants. The average firing rates 


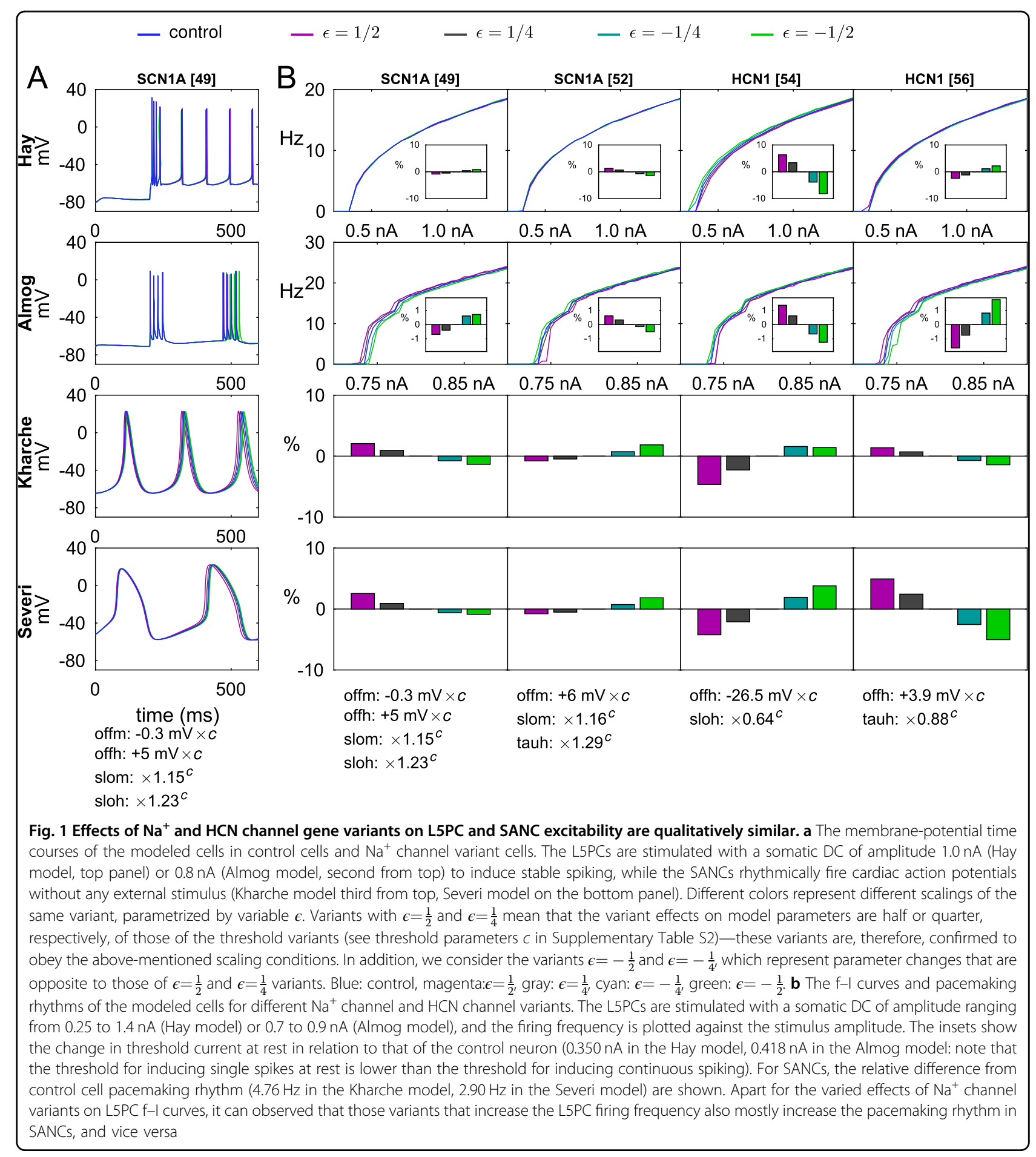

and pacemaking rates of $\mathrm{Ca}^{2+}$ channel and transporter variants were anticorrelated with a correlation coefficient -0.53 to -0.79 , while the corresponding correlation coefficients for $\mathrm{Na}^{+}$and $\mathrm{HCN}$ channel variants were 0.47-0.78 (see Table 2). Exceptions to this trend are discussed in Supplementary information, and the reasons for them are illustrated in Supplementary Figs. S5, S6, S7, and S8, where the time courses of different current species are plotted for control and variant neurons.

The difference in variant effects between L5PC and SANC models is caused by differences in downstream effects of the $\mathrm{Ca}^{2+}$ currents. All four models describe 


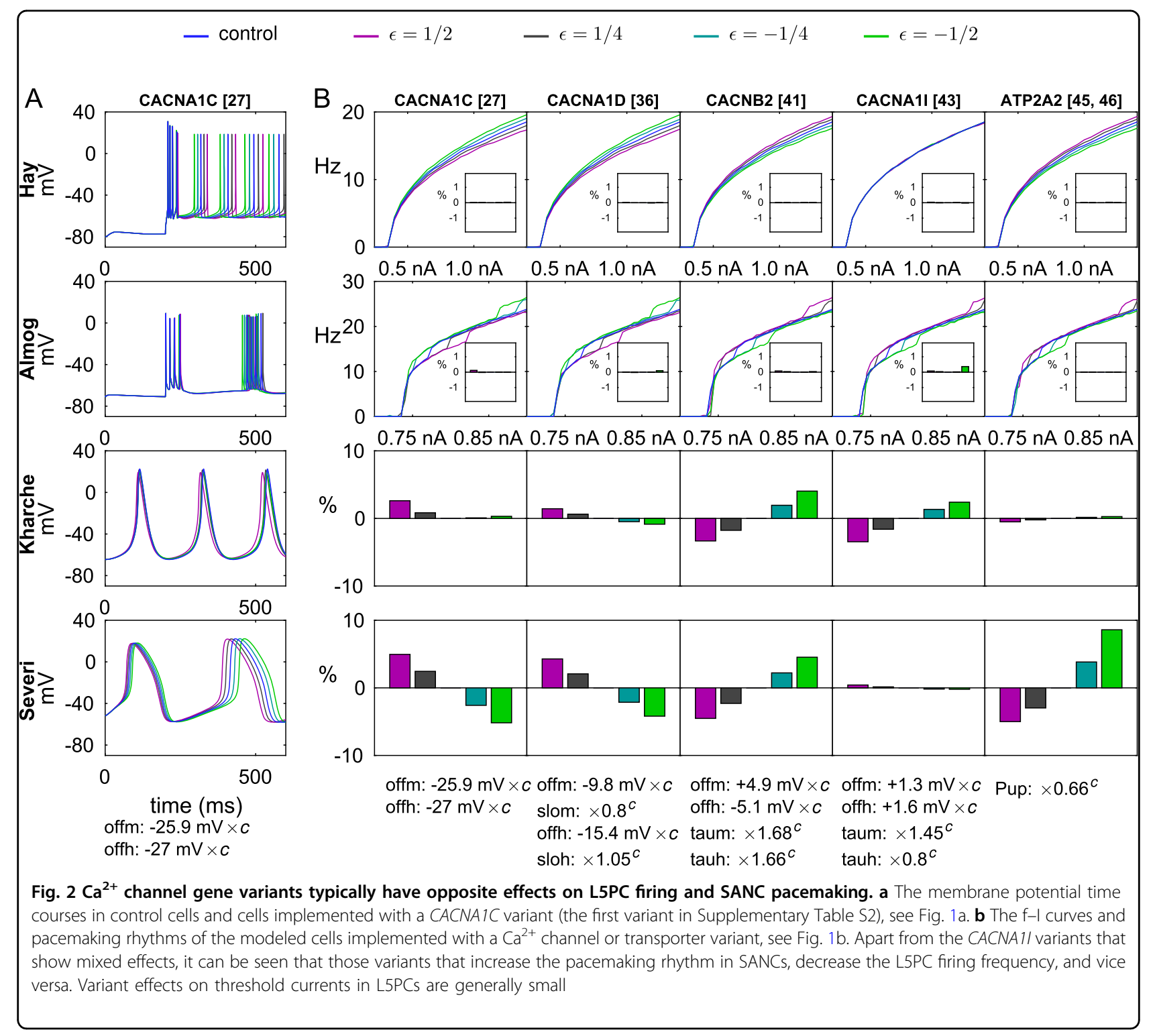

certain aspects of how the intracellular $\left[\mathrm{Ca}^{2+}\right]$, increased by the current influx through the $\mathrm{Ca}^{2+}$ channels, affects the function of other transmembrane ion channels or exchangers. In the L5PC models, increased intracellular $\left[\mathrm{Ca}^{2+}\right]$ activates the $\mathrm{Ca}^{2+}$-dependent $\mathrm{K}^{+}$channels, i.e., the SK channels (and BK channels in the Almog model) that are hyperpolarizing. These channels are traditionally absent from the SANC models, and while recent evidence suggests they may contribute to sinus-node electrophysiology ${ }^{58,59}$, a well-recognized characteristic of SANC function is that enhanced $\mathrm{Ca}^{2+}$ cycling is an important contributor to increased pacemaking frequency both ex vivo and in vivo ${ }^{60}$.

\section{Changes in SANC excitability affect signal propagation}

We implemented a simple 1-dimensional model of interconnected SANCs to analyze the effects of the variants on signal conduction. The SANCs were identical, and were connected to each other with a diffusion constant of $6 \times 10^{4} \mathrm{\mu m}^{2} / \mathrm{ms}$ (as in ref. 61). In this 1-dimensional model, the SANC components were first voltage-clamped to a hyperpolarized membrane potential $(-64 \mathrm{mV})$, and then a fraction of them was clamped to a depolarized membrane potential $(+23 \mathrm{mV})$, and the conduction of this pulse of activation across the SANCs could be observed. Supplementary Fig. S9 shows that subtle variants of $\mathrm{Ca}^{2+}$ channel genes could slightly affect the signal conduction velocity along the sinoatrial node (SAN), and an overview of results for all variants is shown 


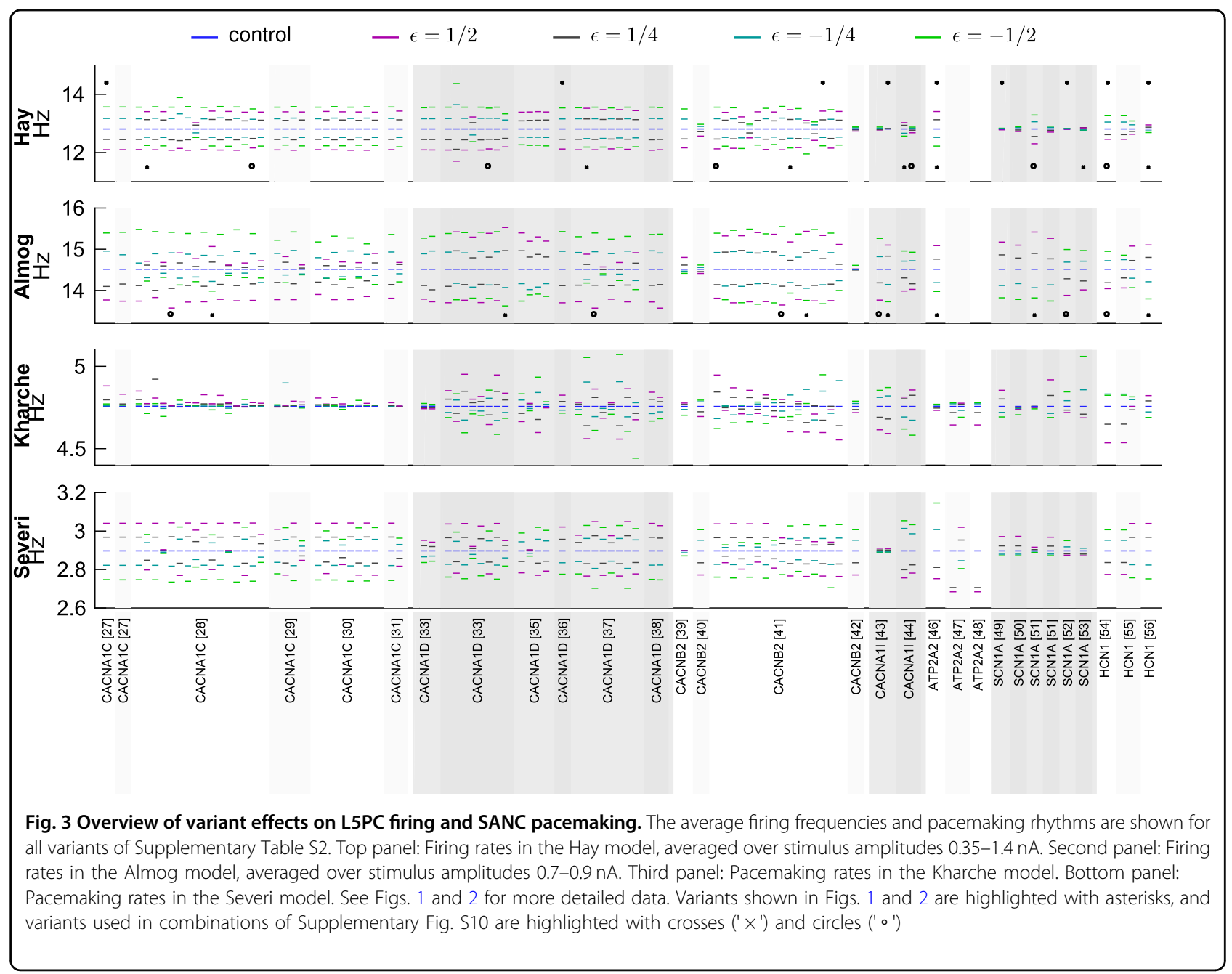

in Supplementary Fig. S11. This propagation is altogether rapid, and, therefore, unlikely to meaningfully alter the speed or sequence of cardiac activation. However, these subtle changes may have larger effects in multiple dimensions and particularly in determining the ability of the SAN to provide sufficient current to activate the surrounding tissue. To test this hypothesis, we applied our variants in a 2-dimensional model including SAN and surrounding atrial tissue. In this experiment, we modeled the SAN tissue using the Severi model and the atrial tissue using the model of ref. 62. We implemented the $C A C$ NA1C variant ${ }^{27}$ (the first entry of Supplementary Table S2) in the SAN tissue using the scaling threshold parameter $c=0.102$. Figure 4 shows the initiation and propagation of cardiac action potentials in this composite 2-dimensional model tissue both for control and variant cases. An interesting finding is shown in Fig. 4b, where the $\epsilon=\frac{1}{2}$ variant caused failure of a premature SAN beat to propagate into the atrial tissue when a large SAN (radius $r=0.4 \mathrm{~cm}$ ) was used. This in turn resulted in an increased beat-to-beat interval, which if frequent enough, would manifest as increased R-R variability and could be clinically identified as an SAN dysfunction.

Figure 4a shows that when a small SAN (radius $r=0.27$ $\mathrm{cm})$ was combined with the $\epsilon=-\frac{1}{2}$ variant, spontaneous SAN activity was silenced. In this case the electrotonic load of the surrounding atrial tissue prevents the current generated by the SANCs from being sufficient to generate a propagating depolarization wave, and thus pacemaking ceases. Such a sharp loss of sinus excitation would be associated with severe (class 1) SAN dysfunction. In an intermediate-sized SAN (radius $r=0.34 \mathrm{~cm}$ ), by contrast, all variants show a stable pacemaking (data not shown).

\section{Discussion}

In this work, using computational modeling we showed how subtle genetic variants of SCZ-associated genes can cause comorbid effects in neuronal and cardiac function. We used models of L5PCs and SANCs due to the biological significance of these cells (in cortical information processing and heart beat initiation, respectively), the high level of biophysical detail with which they are described, 
Table 2 Firing frequencies of L5PCs and pacemaking frequencies of SANCs are correlated for $\mathrm{Na}^{+}$channel and HCN1 variants, but anticorrelated for $\mathrm{Ca}^{2+}$ channel and $\mathrm{Ca}^{2+}$ transporter variants

(A) $\mathrm{Na}^{+}$and $\mathrm{HCN}$ channel variants

\begin{tabular}{lll}
\hline & Hay & Almog \\
\hline Kharche & 0.4965 & 0.7477 \\
Severi & 0.5031 & 0.7818
\end{tabular}

(B) $\mathrm{Ca}^{2+}$ channel and transporter variants

\begin{tabular}{lll}
\hline & Hay & Almog \\
\hline Kharche & -0.5168 & -0.5971 \\
Severi & -0.7700 & -0.7905 \\
& & \\
\hline
\end{tabular}

(C) All variants

\begin{tabular}{lllll}
\hline & Hay & Almog & Kharche & Severi \\
\hline Hay & 1 & 0.6896 & -0.4364 & -0.7223 \\
Almog & 0.6896 & 1 & -0.4233 & -0.6501 \\
Kharche & -0.4364 & -0.4233 & 1 & 0.6645 \\
Severi & -0.7223 & -0.6501 & 0.6645 & 1 \\
\hline
\end{tabular}

The table shows the correlation coefficients between the firing or pacemaking frequencies of $\epsilon=-\frac{1}{4}$ variants (see Fig. 3), as predicted by the different models. A: Only data from SCN1A and HCN1 variants included. B: Only data from CACNA1C, CACNA1D, CACNB2, CACNA1I and ATP2A2 variants included. C: All variants included. The variants that were not applicable for L5PC models (see the Supplementary Table S2 entries corresponding to studies ${ }^{47,48}$ ) were omitted. The relatively strong anticorrelations between L5PC and SANC model data in (C) reflect the fact that majority (80) of the variants (94 in total) operated on $\mathrm{Ca}^{2+}$ channels and transporters

and the similarities in expression of the genes studied here. We showed that small changes in the parameters governing the voltage-dependence and time constants of activation and inactivation of different ion channels caused observable effects in both L5PC and SANC function (Figs. 1-3). In the case of $\mathrm{Ca}^{2+}$ channel gene variants, these changes typically had opposite effects on cell excitability in L5PCs compared to SANCs (higher L5PC firing frequency $\leftrightarrow$ lower SANC pacemaking frequency, see Fig. 2), while in the case of $\mathrm{Na}^{+}$or $\mathrm{HCN}$ channel variants, the effects were mostly similar (higher L5PC firing $\leftrightarrow$ frequency higher SANC pacemaking frequency, see Fig. 1). Our framework is well suited to studying polygenic effects, which is especially important in SCZ: we showed that combinations of subtle variants of different genes can have a large effect on cell excitability (Supplementary Fig. S10). These results are, to our knowledge, the first findings from a polygenic analysis of the pleiotropic effects of genetic variants on neural and cardiac functions.
Our result showing that the gain-of-function $\mathrm{Ca}^{2+}$ channel variants (i.e., variants that increase $\mathrm{Ca}^{2+}$ currents) increase the excitability of SANCs but decrease the excitability of L5PCs, is in line with previous studies. For SANCs, early indirect experimental evidence (e.g., in ref. 63) indicated that larger $\mathrm{Ca}^{2+}$ currents (mediated by an increase in extracellular $\left[\mathrm{Ca}^{2+}\right]$ ) caused faster SAN pacemaking. More recent studies showed that a variety of manipulations that increase whole-cell $\mathrm{Ca}^{2+}$ load also increase SANC pacing frequency, and that their common mechanistic link is spontaneous sarco-endoplasmic reticulum $\mathrm{Ca}^{2+}$ release, which accelerates early SANC depolarization due to $\mathrm{Na}^{+}-\mathrm{Ca}^{2+}$ exchange ${ }^{60}$. The source of SANC pacing is, however, still under debate ${ }^{64}$. The contexts and molecular players determining the importance of these intracellular $\mathrm{Ca}^{2+}$ fluxes remain hotly debated, but there is little doubt that gain-of-function effects in SANC $\mathrm{Ca}^{2+}$ channels results in more rapid beating due either to direct depolarization or secondarily to $\mathrm{Ca}^{2+}$ release from the sarco-endoplasmic reticulum.

For L5PCs, there are numerous experimental studies analyzing the medium-duration after-hyperpolarization (mAHP) current, which is mediated by SK channels and is strong in $\mathrm{L} \mathrm{PCs}{ }^{65}$, but its $\mathrm{Ca}^{2+}$ channel-dependent inhibitory effect on neuron firing has rarely been compared with the direct excitatory effect of the $\mathrm{Ca}^{2+}$ channels. In ref. 66, the effect of blockade of L-type $\mathrm{Ca}^{2+}$ channels on EPSP amplitude was non-significant (albeit slightly weakening the excitability) compared to control. By contrast, computational studies of L5PCs repeatedly predicted that a decrease in current through $\mathrm{Ca}^{2+}$ channels increase the L5PC excitability (due to the consequent decrease in $\mathrm{Ca}^{2+}$-dependent $\mathrm{K}^{+}$current), and vice versa. In addition to the present work, this was concluded in our earlier work ${ }^{16}$ and in an independent study employing a network of L5PCs ${ }^{67}$, where the blockade of voltage-gated $\mathrm{Ca}^{2+}$ channels (especially of those located at the soma) increased the network excitability. Confirming and extending these results may require a spatially detailed neuron model and an extended description of $\mathrm{Ca}^{2+}$ dynamics (cf. ${ }^{65,68}$ ).

Apart from L5PCs, the SK-Ca ${ }^{2+}$ current coupling that reverses the output gain of the $\mathrm{Ca}^{2+}$ currents has been experimentally observed in other types of neurons ${ }^{69}$. These data could be used to validate the results obtained from our method when applied to other cell types. L5PCs are of particular interest among the types of neuron in the brain due to their role as an integrator of sensory feedforward and cortical feed-back information ${ }^{17}$. However, future work should address these research questions in other types of neurons as well, as made possible by the increasing availability of biophysical neuron models ${ }^{70}$, and models of other pacemaker cells and myocytes in the heart. 


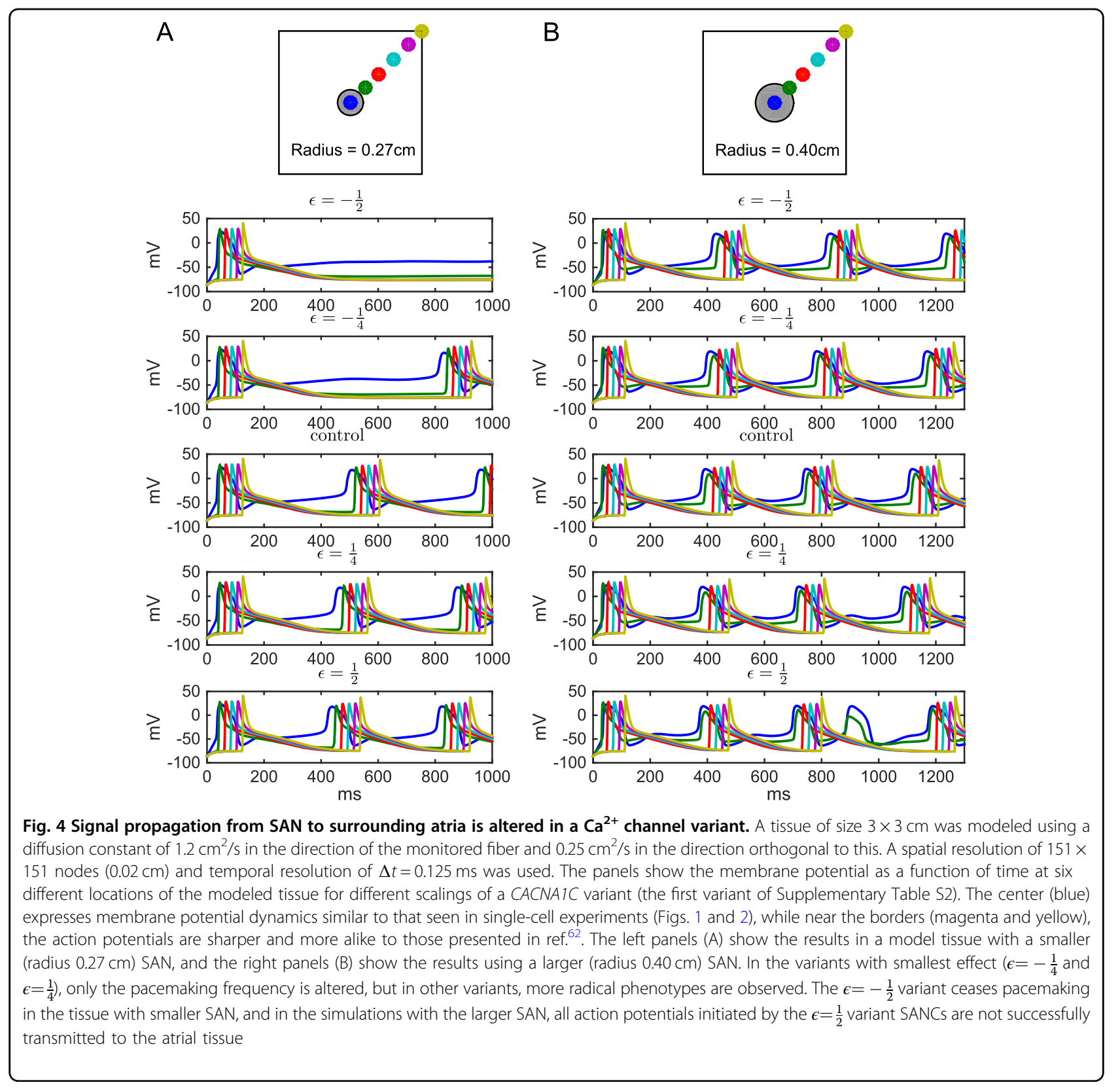

The differences in the levels of biophysical detail between the applied models prevent a consistent use of some of our variants. $\mathrm{Ca}^{2+}$ dynamics were described in more detail in the SANC models than in the L5PC models, which reflects the known importance of $\mathrm{Ca}^{2+}$ cycling in SANC function and allowed for more comprehensive analysis of SERCA (encoded by ATP2A2) variants and their effects on pacemaking. None of the models, however, takes into account nanoscale $\mathrm{Ca}^{2+}$ release events, which may crucially affect the cell electrophysiology ${ }^{71}$. The analyses of genetic effects of $A T P 2 A 2$ were restricted in L5PC models to one variant ${ }^{45,46}$, whose functional effects had previously been measured both in terms of SERCA uptake and cytosolic $\mathrm{Ca}^{2+}$ transients. Of these quantities, the effect on SERCA uptake could be directly applied to SANC models, while the effect on cytosolic $\mathrm{Ca}^{2+}$ transients could be applied to L5PC model parameters. Note, however, that the latter effect might be highly dependent on the cell type-the experiments of ${ }^{46}$ were carried out in myocytes. There is a trend toward the development of increasingly detailed biophysical neuron models and hence a L5PC model incorporating the functions of the endoplasmic reticulum (ER), SERCA pump, and relevant $\mathrm{Ca}^{2+}$ signaling 
molecules could be expected in the near future ${ }^{72}$. Using such models would mitigate the above-mentioned limitation in our approach.

The $I_{f}$ current has notably different characteristics in the two SANC models. In the Kharche model, the description of the $I_{f}$ current was formulated for $H C N 4$-based currents, which have a very negative half-activation potential $(-106.8 \mathrm{mV}$ in the Kharche model) and a less steep slope $(16.3 \mathrm{mV})$. By contrast, in the Severi model the $I_{f}$ current had a much higher half-activation potential $(-52.5 \mathrm{mV})$ and a steeper slope $(9.0 \mathrm{mV})$. The choices concerning the $I_{f}$ current in the Severi model are based on experiments made on rabbit SANCs where expression of both HCN1 and HCN4 were found ${ }^{21,73,74}$, while the Kharche model refers to experiments made on mouse SANCs where expression of only HCN4 was observed ${ }^{20,75}$. However, other studies found $H C N 1$ expression also in mouse $\mathrm{SANCs}^{76,77}-\mathrm{HCN1}$ is also expressed in human SANCs ${ }^{78}$. Thus, the contribution of $H C N 1$ variants to SANC electrophysiology can be expected in both mice and humans, the latter contribution being an important assumption underlying our study.

Despite the differences in the voltage-dependencies of the $I_{f}$ current inactivation, both SANC models agree on the fact that the current is most of the time depolarizing (reversal potentials were approximately $-24 \mathrm{mV}$ and -4 $\mathrm{mV}$ in the Kharche and Severi models, respectively). Moreover, the (depolarizing) amplitude of the $I_{f}$ current is very similar in the two models $(0.006 \mathrm{nA}$ in Kharche model and $0.0067 \mathrm{nA}$ in the Severi model, data not shown). Accordingly, the effects of the $\mathrm{HCN}$ channel variants on the SANC pacemaking were qualitatively similar in the two models, as shown in Figs. 1 and 3, and Supplementary Fig. S2 (an exception is the second-to-last variant of Fig. 3, whose effects on L5PC models were nonanalogous as well, as discussed above).

Our framework is based on downscaling the electrophysiological effects of experimentally studied genetic variants, and as a theoretical approach, it has its limitations. It is not known whether the SCZ-associated SNPs in voltage-dependent ion channel-encoding genes have a measurable effect on the voltage-dependence and kinetics of the underlying channel or not. Furthermore, the magnitudes of the variant effects depend on the conditions used for downscaling the variants, and due to the non-linearity of the neuron models, these conditions may have to be adjusted for each operated model separately to ensure that a single (downscaled) variant does not totally change any fundamental aspect of the cell functionality. Nevertheless, the downscaling framework shows promise as a tool for studying interactions of modest genetic effects, and it can be extended to new cell types and classes of genes. Another limitation of our study stems from the biophysical details that are missing from our models. Like the vast majority of the neuron and cardiac cell models of today, our models do not allow examining the effects of certain biological phenomena controlling the function of ion channels, such as phosphorylation, spatial affinity, or heterogeneous subunit compositions. Extending the models to capture some of these biophysical details would be useful especially in the study of SCZ, as the risk of SCZ has been associated with alterations in not only genes controlling intrinsic electrogenesis (voltage-gated ion channels) and neurotransmission (synaptic ion channels), but also those controlling the calcium signaling machinery affecting the two through protein phosphorylation ${ }^{79}$. Meanwhile, novel tools in neuroinformatics, such as the automated large-scale classification of ion channel models as presented in ref. 80, could help in comparing the modeled effects of genetic variants between different neuron models and making predictions of the cellular functions in modified baseline conditions such as altered temperatures.

Our results shed light on the correlations between neuronal and cardiac phenotypes in SCZ patients. Due to the complexity of clinical manifestations of SCZ, the neural underpinnings of the symptoms are not well understood, but there is a generic hypothesis of SCZ being a disorder of cortical excitability ${ }^{81,82}$. To this end, altered L5PC excitability has been proposed as a contributor to the observed SCZ symptoms and phenotypes, such as hallucinations ${ }^{16,17}$. Altered synaptic function has also been associated to SCZ (suggested both by GWASs and imaging studies), but is out of the scope of the present study. The L5PC functions studied here were restricted to responses to somatic DC-for a more detailed analysis of the effects of the variants on integration of synaptic inputs, we refer to our earlier work ${ }^{16}$. In this work, we showed that the same subtle genetic variants that altered the L5PC excitability, also altered SANC pacemaking frequency and rate of propagation of the cardiac action potential. While these deviations are specific to pacemaker tissues in the heart, similar pleiotropic effects occurring in the ventricular myocardium could prolong the action potential or increase dispersion of repolarization, and thus be associated with the prolonged QT interval observed in drug-free $\mathrm{SCZ}$ patients ${ }^{83}$. An interesting observation is that the variants may affect the probability of successful signal propagation from the SAN to the surrounding atrial tissue, as shown in Fig. $4 \mathrm{~b}$. In these simulations, the variant effects were only implemented in the sinoatrial tissue-were they present also in the atrial tissue, the observed effects on signal propagation could be larger. When this propagation failure is complete, the condition is termed third degree sinoatrial block. More subtle effects include a sporadic block that simply alters P-P variability (and therefore R-R variability) similar to that shown in Fig. 4b. By inducing irregular long 
pauses in ventricular activation, this type of behavior may contribute to the emergence of ventricular tachycardias, particularly the torsade de pointes that accompanies both acquired and congenital long QT syndrome ${ }^{84,85}$. This form of polymorphic ventricular tachycardia is also associated with the drug-induced long QT syndrome that is a major contraindication of many antipsychotic drugs ${ }^{9}$.

Our results provide interesting views on the dual effects of SNP-like effects on neuronal and cardiac excitability. While the rare variants of ion-channel-encoding genes often have a disabling or even life-threatening phenotypic effect, the effects of common variants may be subtle and highly specific to certain types of tissue or cell type. The work at hand illustrates the polygenic effects of SCZassociated genes by borrowing the functional genomic data from gene variants that implicate other, typically larger, phenotypic consequences, and studying the cellular functions under downscaled variant effects. These results provide an important viewpoint on the polygenic alterations of neuronal and cardiac excitability, but eventually, the electrophysiological consequences of the common, SCZ-associated SNPs should be assessed as well. Novel automated cell-patching methods ${ }^{86,87}$ could help in this vast task.

To conclude, the current findings support the use of a polygenic mathematical modeling approach to understand more of the pathobiology related to the GWASrevealed SCZ-associated loci. Our results suggest overlapping but non-identical mechanisms through which subtle SNP-like variants of ion-channel and calciumtransporter-encoding genes modulate the intrinsic excitability of neurons and heart cells. This may explain some of the comorbidity between cardiac disease and SCZ, and could facilitate development of antipsychotic drugs with fewer cardiac side-effects.

\section{Acknowledgements}

NOTUR resources were used for the simulations. Funding: $\mathrm{NIH}$ grant 5 R01 EB000790-10, EC-FP7 grant 604102 ("Human Brain Project"), Research Council of Norway $(216699,248778,223273,249711$, and 248828), South East Norway Health Authority (2017-112), and KG Jebsen Stiftelsen.

\begin{abstract}
Author details
'NORMENT, KG Jebsen Centre for Psychosis Research, Institute of Clinical Medicine, University of Oslo, Oslo, Norway. ${ }^{2}$ Simula Research Laboratory and Center for Cardiological Innovation, Oslo, Norway. ${ }^{3}$ Multimodal Imaging Laboratory, UC San Diego, La Jolla, CA, USA. ${ }^{4}$ Department of Neurosciences, University of California San Diego, La Jolla, CA, USA. ${ }^{5}$ Department of Radiology, University of California, San Diego, La Jolla, CA, USA. ${ }^{6}$ Department of Mathematical Sciences and Technology, Norwegian University of Life Sciences, Ås, Norway. ${ }^{7}$ Department of Physics, University of Oslo, Oslo, Norway. ${ }^{8}$ Division of Mental Health and Addiction, Oslo University Hospital, Oslo, Norway
\end{abstract}

\section{Competing interests}

The authors declare that they have no competing financial interests.

\section{Supplementary information}

The online version of this article (https://doi.org/10.1038/s41398-017-0007-4) contains supplementary material.

Received: 6 March 2017 Revised: 7 July 2017 Accepted: 14 July 2017

Published online: 17 November 2017

\section{References}

1. Ripke, S. et al. Genome-wide association analysis identifies 13 new risk loci for schizophrenia. Nat. Genet. 45, 1150-1159 (2013).

2. Ripke, S. et al. Biological insights from 108 schizophrenia-associated genetic loci. Nature 511, 421-427 (2014).

3. Saha, S., Chant, D. \& McGrath, J. A systematic review of mortality in schizophrenia: Is the differential mortality gap worsening over time? Arch. Gen. Psychiatr. 64, 1123-1131 (2007).

4. Laursen, T. M., Munk-Olsen, T. \& Vestergaard, M. Life expectancy and cardiovascular mortality in persons with schizophrenia. Curr. Opin. Psychiatr. 25, 83-88 (2012).

5. Brown, S. Excess mortality of schizophrenia. A meta-analysis. Br. J. Psychiatr. 171, 502-508 (1997).

6. Ryan, M. C. \& Thakore, J. H. Physical consequences of schizophrenia and its treatment: The metabolic syndrome. Life Sci. 71, 239-257 (2002).

7. Ray, W. A., Chung, C. P., Murray, K. T., Hall, K. \& Stein, C. M. Atypical antipsychotic drugs and the risk of sudden cardiac death. N. Eng. J. Med. 360, 225-235 (2009).

8. Reilly, J., Ayis, S., Ferrier, I., Jones, S. \& Thomas, S. QTc-interval abnormalities and psychotropic drug therapy in psychiatric patients. The Lancet 355, 1048-1052 (2000).

9. Glassman, A. H. \& Bigger, J. T. Jr Antipsychotic drugs: Prolonged QTc interval, torsade de pointes, and sudden death. Am. J. Psychiatr. 158, 1774-1782 (2001).

10. Sotoodehnia, N. et al. Common variants in 22 loci are associated with QRS duration and cardiac ventricular conduction. Nat. Genet. 42, 1068-1076 (2010).

11. Arking, D. E. et al. Genetic association study of QT interval highlights role for calcium signaling pathways in myocardial repolarization. Nat. Genet. 46, 826-836 (2014).

12. Lehnart, S. E. et al. Leaky $\mathrm{Ca}^{2+}$ release channel/ryanodine receptor 2 causes seizures and sudden cardiac death in mice. J. Clin. Invest. 118, 2230 (2008).

13. Splawski, I. et al. Severe arrhythmia disorder caused by cardiac L-type calcium channel mutations. Proc. Natl Acad. Sci. 102, 8089-8096 (2005).

14. Markram, H. The Human Brain Project. Sci. Am. 306, 50-55 (2012).

15. Iop L. Conference Papers in Science. Vol. 2014, p. 369246 (Hindawi Publishing Corporation, 2014)

16. Mäki-Marttunen, T. et al. Functional effects of schizophrenia-linked genetic variants on intrinsic single-neuron excitability: A modeling study. Biol. Psychiatr.: Cogn. Neurosci. Neuroimaging. 1, 49-59 (2016).

17. Larkum, M. A cellular mechanism for cortical associations: An organizing principle for the cerebral cortex. Trends Neurosci. 36, 141-151 (2013).

18. Hay, E., Hill, S., Schürmann, F., Markram, H. \& Segev, I. Models of neocortical layer $5 \mathrm{~b}$ pyramidal cells capturing a wide range of dendritic and perisomatic active properties. PLoS Comput. Biol. 7, e1002107 (2011).

19. Almog, M. \& Korngreen, A. A quantitative description of dendritic conductances and its application to dendritic excitation in layer 5 pyramidal neurons. J. Neurosci. 34, 182-196 (2014).

20. Kharche, S., Yu, J., Lei, M. \& Zhang, H. A mathematical model of action potentials of mouse sinoatrial node cells with molecular bases. Am. J. Physiol. Heart Circ. Physiol. 301, H945-H963 (2011).

21. Severi, S., Fantini, M., Charawi, L. A. \& DiFrancesco, D. An updated computational model of rabbit sinoatrial action potential to investigate the mechanisms of heart rate modulation. J. Physiol. 590, 4483-4499 (2012).

22. Gottesman, I. I. \& Shields, J. A polygenic theory of schizophrenia. Proc. Natl Acad. Sci. 58, 199 (1967).

23. Purcell, S. M. et al. Common polygenic variation contributes to risk of schizophrenia and bipolar disorder. Nature 460, 748-752 (2009).

24. Sundnes J., et al. Computing the Electrical Activity in the Heart. Vol. 1 (Springer Science \& Business Media, 2007).

25. Logg A., Mardal K. A., Wells G. Automated Solution of Differential Equations by the Finite Element Method: The FEniCS Book. Vol. 84. (Springer Science \& Business Media, 2012). 
26. Li, P., Lines, G. T., Maleckar, M. M. \& Tveito, A. Mathematical models of cardiac pacemaking function. Front. Phys. 1, 20 (2013).

27. Kudrnac, M. et al. Coupled and independent contributions of residues in IS6 and IIS6 to activation gating of CaV1.2. J. Biol. Chem. 284, 12276-12284 (2009).

28. Depil, $\mathrm{K}$. et al. Timothy mutation disrupts the link between activation and inactivation in Cav1.2 protein. J. Biol. Chem. 286, 31557-31564 (2011).

29. Hohaus, A. et al. Structural determinants of L-type channel activation in segment IIS6 revealed by a retinal disorder. J. Biol. Chem. 280, 38471-38477 (2005).

30. Stary, A. et al. Molecular dynamics and mutational analysis of a channelopathy mutation in the IIS6 helix of Cav1.2. Channels 2, 216-223 (2008).

31. Tang, Z. Z. et al. Transcript scanning reveals novel and extensive splice variations in human L-type voltage-gated calcium channel, Cav1.2 a1 subunit. J. Biol. Chem. 279, 44335-44343 (2004).

32. Tan, B. Z. et al. Functional characterization of alternative splicing in the C terminus of L-type CaV1. 3 channels. J. Biol. Chem. 286, 42725-42735 (2011).

33. Bock, G. et al. Functional properties of a newly iden- tified C-terminal splice variant of Cav1.3 L-type Ca2+channels. J. Biol. Chem. 286, 42736-42748 (2011).

34. Zhang, Q. et al. Expression and roles of Cav1.3 (a1D) L-type Ca2+channel in atrioventricular node automaticity. J. Mol. Cell. Cardiol. 50, 194-202 (2011).

35. Pérez-Alvarez, A., Hernández-Vivanco, A., Caba-González, J. C. \& Albillos, A. Different roles attributed to Cav1 channel subtypes in spontaneous action potential firing and fine tuning of exocytosis in mouse chromaffin cells. J. Neurochem. 116, 105-121 (2011).

36. Pinggera, A. et al. CACNA1D de novo mutations in autism spectrum disorders activate Cav1.3 L-type calcium channels. Biol. Psychiatr. 77, 816-822 (2015).

37. Azizan, E. A. et al. Somatic mutations in ATP1A1 and CACNA1D underlie a common subtype of adrenal hypertension. Nat. Genet. 45, 1055-1060 (2013).

38. Lieb, A., Scharinger, A., Sartori, S., Sinnegger-Brauns, M. J. \& Striessnig, J. Structural determinants of CaV1. 3 L-type calcium channel gating. Channels $\mathbf{6}$, 197-205 (2012).

39. Cordeiro, J. M. et al. Accelerated inactivation of the L-type calcium current due to a mutation in CACNB2b underlies Brugada syndrome. J. Mol. Cell. Cardiol. 46, 695-703 (2009).

40. Massa, E., Kelly, K. M., Yule, D. I., MacDonald, R. L. \& Uhler, M. D. Comparison of fura-2 imaging and electrophysiological analysis of murine calcium channel $a$ 1 subunits coexpressed with novel $\beta 2$ subunit isoforms. Mol. Pharmacol. 47, 707-716 (1995).

41. Link, S. et al. Diversity and developmental expression of L-type calcium channel $\beta 2$ proteins and their influence on calcium current in murine heart. J. Biol. Chem. 284, 30129-30137 (2009).

42. Hu, D. et al. Dual variation in SCN5A and CACNB2b underlies the development of cardiac conduction disease without Brugada syndrome. Pacing Clin. Electrophysiol. 33, 274-285 (2010).

43. Murbartián, J., Arias, J. M. \& Perez-Reyes, E. Functional impact of alternative splicing of human T-type Cav3.3 calcium channels. J. Neurophysiol. 92 3399-3407 (2004).

44. Gomora, J. C., Murbartian, J., Arias, J. M., Lee, J. H. \& Perez-Reyes, E. Cloning and expression of the human T-type channel Cav3.3: insights into prepulse facilitation. Biophys. J. 83, 229-241 (2002).

45. Periasamy, M. et al. Impaired cardiac performance in heterozygous mice with a null mutation in the sarco (endo) plasmic reticulum Ca2+-ATPase isoform 2 (SERCA2) gene. J. Biol. Chem. 274, 2556-2562 (1999).

46. Ji, Y. et al. Disruption of a single copy of the SERCA2 gene results in altered Ca2+homeostasis and cardiomyocyte function. J. Biol. Chem. 275 38073-38080 (2000).

47. Dode, L. et al. Dissection of the functional differences between sarco (endo) plasmic reticulum Ca2+-ATPase (SERCA) 1 and 2 isoforms and characterization of Darier disease (SERCA2) mutants by steady-state and transient kinetic analyses. J. Biol. Chem. 278, 47877-47889 (2003).

48. Ahn, W., Lee, M. G., Kim, K. H. \& Muallem, S. Multiple effects of SERCA2b mutations associated with Darier's disease. J. Biol. Chem. 278, 20795-20801 (2003).

49. Cestèle, S. et al. Self-limited hyperexcitability: Functional effect of a familial hemiplegic migraine mutation of the Nav1.1 (SCN1A) Na+channel. J. Neurosci. 28, 7273-7283 (2008).

50. Vanmolkot, K. R. et al. The novel p.L1649Q mutation in the SCN1A epilepsy gene is associated with familial hemiplegic migraine: Genetic and functional studies. Hum. Mutat. 28, 522-522 (2007).

51. Volkers, L. et al. Nav1.1 dysfunction in genetic epilepsy with febrile seizuresplus or Dravet syndrome. Eur. J. Neurosci. 34, 1268-1275 (2011).
52. Cestèle, S. et al. Divergent effects of the T1174S SCN1A mutation associated with seizures and hemiplegic migraine. Epilepsia 54, 927-935 (2013).

53. Mantegazza, M. et al. Identification of an Nav1.1 sodium channel (SCN1A) lossof-function mutation associated with familial simple febrile seizures. Proc. Natl Acad. Sci. 102, 18177-18182 (2005).

54. Ishii, T. M., Nakashima, N. \& Ohmori, H. Tryptophan-scanning mutagenesis in the S1 domain of mammalian HCN channel reveals residues critical for voltage-gated activation. J. Physiol. 579, 291-301 (2007).

55. Lesso, H. \& Li, R. A. Helical secondary structure of the external S3-S4 linker of pacemaker $(\mathrm{HCN})$ channels revealed by site-dependent perturbations of activation phenotype. J. Biol. Chem. 278, 22290-22297 (2003).

56. Wemhöner, K. et al. An N-terminal deletion variant of HCN1 in the epileptic WAG/Rij strain modulates HCN current densities. Front. Mol. Neurosci. 8, 63 (2015).

57. Lee, S. H. et al. Estimating the proportion of variation in susceptibility to schizophrenia captured by common SNPs. Nat. Genet. 44, 247-250 (2012).

58. Chen, W. T. et al. Apamin modulates electrophysiological characteristics of the pulmonary vein and the sinoatrial node. Eur. J. Clin. Invest. 43, 957-963 (2013).

59. Lai, M. H. et al. BK channels regulate sinoatrial node firing rate and cardiac pacing in vivo. Am. J. Physiol. Heart Circ. Physiol. 307, H1327-H1338 (2014).

60. Lakatta, E. G., Maltsev, V. A. \& Vinogradova, T. M. A coupled system of intracellular Ca2+ clocks and surface membrane voltage clocks controls the timekeeping mechanism of the heart's pacemaker. Circ. Res. 106, 659-673 (2010).

61. Zhang, $\mathrm{H}$. et al. Mathematical models of action potentials in the periphery and center of the rabbit sinoatrial node. Am. J. Physiol. Heart Circ. Physiol. 279, H397-H421 (2000)

62. Koivumäki, J. T., Korhonen, T. \& Tavi, P. Impact of sarcoplasmic reticulum calcium release on calcium dynamics and action potential morphology in human atrial myocytes: a computational study. PLoS Comput. Biol. 7, e1001067 (2011).

63. Seifen, E., Schaer, $H$. \& Marshall, J. Effect of calcium on the membrane potentials of single pacemaker fibres and atrial fibres in isolated rabbit atria. Nature 202, 1223-1224 (1964).

64. Lakatta, E. G. \& DiFrancesco, D. What keeps us ticking, a funny current, a calcium clock, or both? J. Mol. Cell. Cardiol. 47, 157 (2009).

65. Stocker, M. Ca ${ }^{2+}$-activated K+channels: Molecular determinants and function of the SK family. Nat. Rev. Neurosci. 5, 758-770 (2004).

66. Faber, E. Functional interplay between NMDA receptors, SK channels and voltage-gated Ca2+channels regulates synaptic excitability in the medial prefrontal cortex. J. Physiol. 588, 1281-1292 (2010).

67. Papoutsi, A., Sidiropoulou, K., Cutsuridis, V. \& Poirazi, P. Induction and modulation of persistent activity in a layer $V$ PFC microcircuit model. Front. Neural Circuits 7, 161 (2013).

68. Jones, S. L. \& Stuart, G. J. Different calcium sources control somatic versus dendritic SK channel activation during action potentials. J. Neurosci. 33, 19396-19405 (2013)

69. Simms, B. A. \& Zamponi, G. W. Neuronal voltage-gated calcium channels: Structure, function, and dysfunction. Neuron 82, 24-45 (2014).

70. Markram, $\mathrm{H}$. et al. Reconstruction and simulation of neocortical microcircuitry. Cell 163, 456-492 (2015).

71. Macquaide, N. et al. Ryanodine receptor cluster fragmentation and redistribution in persistent atrial fibrillation enhance calcium release. Cardiovasc. Res. 108, 387-398 (2015).

72. Blackwell, K. Approaches and tools for modeling signaling pathways and calcium dynamics in neurons. J. Neurosci. Methods 220, 131-140 (2013).

73. Altomare, C. et al. Heteromeric HCN1-HCN4 channels: a comparison with native pacemaker channels from the rabbit sinoatrial node. J. Physiol. 549, 347-359 (2003)

74. Barbuti, A., Baruscotti, M. \& Difrancesco, D. The pacemaker current: from basics to the clinics. J. Cardiovasc. Electrophysiol. 18, 342-347 (2007).

75. Liu, J., Dobrzynski, H., Yanni, J., Boyett, M. R. \& Lei, M. Organisation of the mouse sinoatrial node: structure and expression of HCN channels. Cardiovasc. Res. 73 729-738 (2007)

76. Moosmang, $\mathrm{S}$. et al. Cellular expression and functional characterization of four hyperpolarization-activated pacemaker channels in cardiac and neuronal tissues. Eur. J. Biochem. 268, 1646-1652 (2001).

77. Marionneau, $C$. et al. Specific pattern of ionic channel gene expression associated with pacemaker activity in the mouse heart. J. Physiol. 562, 223-234 (2005).

78. Li, N. et al. Molecular mapping of sinoatrial node HCN channel expression in the human heart. Circ. Arrhythm. Electrophysiol. 8, 1219-1227 (2015). p. CIRCEP-115. 
79. Devor, A. et al. Genetic evidence for role of integration of fast and slow neurotransmission in schizophrenia. Mol. Psychiatr. 22, 792-801 (2017).

80. Podlaski, W. F. et al. Mapping the function of neuronal ion channels in model and experiment. elife 6, e22152 (2017).

81. O'Donnell P. Cortical Deficits In Schizophrenia, p. 219-236 (Springer, 2008).

82. Hasan, A., Falkai, P. \& Wobrock, T. Transcranial brain stimulation in schizophrenia: Targeting cortical excitability, connectivity and plasticity. Curr. Med. Chem. 20, 405-413 (2013).

83. Fujii, K. et al. QT is longer in drug-free patients with schizophrenia compared with age-matched healthy subjects. PLOS ONE 9, e98555 (2014).
84. Viskin, S. et al. Mode of onset of torsade de pointes in congenital long QT syndrome. J. Am. Coll. Cardiol. 28, 1262-1268 (1996).

85. Viskin, S. et al. Arrhythmias in the congenital long QT syndrome: How often is torsade de pointes pause dependent? Heart 83, 661-666 (2000).

86. Ranjan R., et al. Automated Biophysical Characterization of the Complete Rat Kvion Channel Family, 2014. Society for Neuroscience Meeting (SfN 2014), Washington, DC, USA. November 15-19, 2014.

87. Kodandaramaiah, S. B., Franzesi, G. T., Chow, B. Y., Boyden, E. S. \& Forest, C. R Automated whole-cell patch-clamp electrophysiology of neurons in vivo. Nat. Methods 9, 585-587 (2012). 